PHYSICAL REVIEW D 95, 025014 (2017)

\title{
Symmetries in superintegrable deformations of oscillator and Coulomb systems: Holomorphic factorization
}

\author{
Tigran Hakobyan ${ }^{*}$ and Armen Nersessian \\ Yerevan State University, 1 Alex Manoogian Street, Yerevan 0025, Armenia and Tomsk Polytechnic \\ University, Lenin Avenue 30, 634050 Tomsk, Russia
}

Hovhannes Shmavonyan

Yerevan Physics Institute, 2 Alikhanian Brothers Street, Yerevan 0036, Armenia

(Received 7 December 2016; published 20 January 2017)

\begin{abstract}
We propose a unified description for the constants of motion for superintegrable deformations of the oscillator and Coulomb systems on $N$-dimensional Euclidean space, sphere, and hyperboloid. We also consider the duality between these generalized systems and present some examples.
\end{abstract}

DOI: $10.1103 /$ PhysRevD.95.025014

\section{INTRODUCTION}

The $N$-dimensional oscillator and Coulomb problem plays a special role among other integrable systems for many reasons. One of the main reasons, for which these models continue to attract permanent interest during the past centuries, is the "maximal superintegrability," i.e., the existence of the maximally possible number, $2 N-1$, of functionally independent constants of motion. The rational Calogero model with the oscillator potential [1] and its generalization associated with arbitrary Coxeter systems [2] is a superintegrable system as well [3]. The oscillator and Coulomb systems admit obvious separation of the radial and angular variables, which is useful for formulating in terms of conformal algebra $\operatorname{so}(1,2) \equiv \operatorname{sl}(2, \mathbb{R})$ defined by the following Poisson bracket relations:

$\left\{\mathcal{H}_{0}, \mathcal{D}\right\}=2 \mathcal{H}_{0}, \quad\left\{\mathcal{H}_{0}, \mathcal{K}\right\}=\mathcal{D}, \quad\{\mathcal{K}, \mathcal{D}\}=-2 \mathcal{K}$.

The generators $\mathcal{H}_{0}, \mathcal{K}, \mathcal{D}$ could be identified, respectively, with the Hamiltonian of some $\mathrm{N}$-dimensional mechanical system, and with the generators of conformal boost and dilatation. This system is usually called "conformal mechanics," and so $(1,2)$ symmetry appears as its dynamical symmetry [4]. The effective "radius" and conjugated momentum are introduced,

$$
r=\sqrt{2 \mathcal{K}}, \quad p_{r}=\frac{\mathcal{D}}{\sqrt{2 \mathcal{K}}}, \quad\left\{p_{r}, r\right\}=1,
$$

and a Casimir of conformal algebra is defined,

$\mathcal{I}=2 \mathcal{H}_{0} \mathcal{K}-\frac{1}{2} \mathcal{D}^{2}:\left\{\mathcal{I}, \mathcal{H}_{0}\right\}=\{\mathcal{I}, \mathcal{K}\}=\{\mathcal{I}, \mathcal{D}\}=0$.

\footnotetext{
*tigran.hakobyan@ysu.am

arnerses@ysu.am

¥shmavonyanhov@gmail.com
}

It is obviously a constant of motion independent of the radial coordinate and momentum, and thus could be expressed via appropriate angular coordinates $\phi_{a}$ and canonically conjugate momenta $\pi_{a}$ which are independent on radial ones: $\mathcal{I}=\mathcal{I}\left(\phi_{a}, \pi_{a}\right)$. In these terms the generators of conformal algebra read

$$
\mathcal{H}_{0}=\frac{p_{r}^{2}}{2}+\frac{\mathcal{I}}{r^{2}}, \quad \mathcal{D}=r p_{r}, \quad \mathcal{K}=\frac{r^{2}}{2} .
$$

Hence, such a separation of angular and radial parts could be defined for any system with dynamical conformal symmetry, and those with additional potentials could be functions of conformal boost $\mathcal{K}$. In particular, such generalized oscillator and Coulomb systems assume adding the potential

$$
V_{\mathrm{osc}}=\omega^{2} \mathcal{K}, \quad V_{\mathrm{Coul}}=-\frac{\gamma}{\sqrt{2 \mathcal{K}}},
$$

so that their Hamiltonian takes the form

$$
\mathcal{H}_{\text {osc } / \text { Coul }}=\frac{p_{r}^{2}}{2}+\frac{\mathcal{I}}{r^{2}}+V_{\text {osc } / \text { Coul }}(r) .
$$

Well-known generalizations of oscillator and Coulomb systems to $N$-dimensional spheres and two-sheet hyperboloids (pseudospheres) [5] can be described in a similar way (see Sec. V).

In Refs. [6,7] a separation of "radial" and "angular" variables has been used for constructing the integrable deformations of oscillator and Coulomb systems [and of their (pseudo)spherical generalizations] via replacement of the spherical part of pure oscillator and Coulomb Hamiltonians [quadratic Casimir of $S O(N)$ algebra] by some other integrable system formulated in terms of the action-angle variables. By analyzing these deformations in terms of action-angle variables, it was found that they are superintegrable iff the spherical part has the form 


$$
\mathcal{I}=\frac{1}{2}\left(\sum_{a=1}^{N-1} k_{a} I_{a}+c_{0}\right)^{2}
$$

with $c_{0}$ as an arbitrary constant and $k_{a}$ as rational numbers. Moreover, it was demonstrated, by the use of the results of Ref. [8], that the angular part of the rational Calogero model belongs to this set of systems. Thus, it was concluded that the rational Calogero model with the Coulomb potential (Calogero-Coulomb system) is a superintegrable system. Besides, superintegrable generalizations of the rational Calogero models with oscillator and Coulomb potentials on the $N$-dimensional spheres and two-sheet hyperboloids have been suggested there. The explicit expressions of their symmetry generators and respective algebras have been given in Refs. $[9,10]$. An integrable two-center generalization of the CalogeroCoulomb systems (and those in the presence of a Stark term, which was called the Calogero-Coulomb-Stark model) has also been revealed [11]. Other superintegrable deformations of the two-dimensional oscillator and Coulomb systems of this kind are known as the Trembley-Turbiner-Wintenitz (TTW) system [12] and the Post-Winternitz (PW) system [13]. They are defined by the Hamiltonians (6) with the angular part given by the Pöschl-Teller system on a circle that is a particular case of the Calogero-oscillator system, and their generalizations to sphere and hyperboloid [6]

$$
\mathcal{I}_{P T}=\frac{p_{\varphi}^{2}}{2}+\frac{k^{2} \alpha^{2}}{\sin ^{2} k \varphi}+\frac{k^{2} \beta^{2}}{\sin ^{2} k \varphi},
$$

where $k$ is an (half-)integer. The superintegrability of these systems was observed initially by numerical simulations, and only later was an analytic expression for the additional constant of motion presented [14]. Initially these systems were invented as new superintegrable models, but soon it was observed that they coincide with the two-dimensional rational Calogero model with the oscillator and Coulomb potential associated with the dihedral group $D_{k}$ [15]. (Super)integrability of their (pseudo)spherical counterparts was noticed in Ref. [6]. Nevertheless, their study has attracted much attention up to now. Among interesting observations on this subject was the so-called "holomorphic factorization" of constants of motions of these systems developed by Ranada [16], which could be viewed as a classical counterpart of the factorization of the Schrödinger operator. In this approach all constants of motion of the two-dimensional oscillator and Coulomb systems were presented as a single complex integral, which was represented as a product of two complex functions: one of the latter involves only angular variables, and the other radial ones and on (8). In our recent paper [17] it was observed that the radial part of this complex function is related to the coordinate parametrizing Klein model Lobachevsky plane so that the $s o(1,2)$ generators define its Killing potentials
(Hamiltonian generators of the isometries of the Kähler structure), while the angular part is related to the angle variable of the Pöschl-Teller Hamiltonian (8), in agreement with Ref. [18]. This allowed them to suggest the extension of that construction to higher-dimensional (super)integrable systems with the oscillator and Coulomb potential.

The goal of this paper is to present holomorphic factorization to the superintegrable generalizations of oscillator and Coulomb systems on $N$-dimensional Euclidean space, sphere, and two-sheet hyperboloid (pseudosphere). For this purpose we parametrize the phase spaces of that system by the complex variable $z=p_{r}+$ $l \sqrt{2 \mathcal{I}} / r$ identifying the radial phase subspace with the Klein model of Lobachevsky plane (compare with Refs. [17,18]), and by the complex variables $u_{a}=$ $\sqrt{I_{a}} \mathrm{e}^{l \Phi_{a}}$ unifying action-angle variables of the angular part of the systems. We formulate, in these terms, the constants of motion of the systems under consideration and calculate their algebra. Besides, we extend to these systems the known oscillator-Coulomb duality transformation.

The paper is organized as follows:

In Sec. II we introduce the appropriate complex coordinates unifying radial and angular variables and formulate the Poisson brackets and generators of conformal algebra in these terms. Then we give the "holomorphic factorization formulation" of the constants of motion of higherdimensional superintegrable conformal mechanics and calculate their algebra.

In Sec. III we formulate in these terms, the higherdimensional superintegrable generalizations of oscillator and Coulomb systems given by (4) and (7) and calculate the algebra of their constants of motion.

In Sec. IV we formulate, in these terms, the well-known oscillator-Coulomb duality transformation.

In Sec. V we extend the results of Sec. II to the systems on the $\mathrm{N}$-dimensional sphere and two-sheet hyperboloid (pseudosphere).

Finally, in Sec. VI we formulate in these terms the special angular part of these systems.

\section{CONFORMAL MECHANICS}

Let us consider the $N$-dimensional conformal mechanics, defined by the canonical symplectic structure $d \mathbf{p} \wedge d \mathbf{x}$ and the Hamiltonian

$\mathcal{H}_{0}=\frac{\mathbf{p}^{2}}{2}+V(\mathbf{x}) \quad$ with $\quad(\mathbf{x} \cdot \nabla) V(\mathbf{x})=-2 V(\mathbf{x})$.

The conformal algebra (1) is generated by the $\mathcal{H}_{0}$ and the generators of dilatation and conformal boost,

$$
\mathcal{D}=\mathbf{p} \cdot \mathbf{x}, \quad \mathcal{K}=\frac{\mathbf{x}^{2}}{2} .
$$

Extracting the radius $r=|\mathbf{x}|$ and its canonically conjugated momentum $p_{r}=\frac{\mathbf{p} \cdot \mathbf{x}}{r}$, we can write these generators in the 
form (4) with $\mathcal{I}=\mathcal{I}\left(\phi_{a}, \pi_{a}\right)$ as the Casimir element of the so $(1,2)$ algebra, depending on the angular coordinates $\varphi_{a}$ and their canonically conjugated momenta $\pi_{a}$. Considered itself as a separate Hamiltonian, $\mathcal{I}$ describes a particle on the $(N-1)$ sphere, moving in the field of the potential $U\left(\phi_{a}\right)=r^{2} V(\mathbf{x})$ (various aspects of these systems were studied in [19]).

To provide the conformal mechanics by integrability property, we choose an integrable angular system, formulated in terms of the action-angle variables,

$$
\mathcal{I}=\mathcal{I}\left(I_{a}\right), \quad \Omega=\sum_{a=1}^{N-1} d I_{a} \wedge d \Phi_{a}, \quad \Phi_{a} \in[0,2 \pi)
$$

Introducing the complex variable $z$, identifying the radial phase subspace with the Klein model of Lobachevsky plane (compare with $[17,18]$ ), and providing complex variables $u_{a}$ unifying the action-angle variables,

$$
\begin{aligned}
z & =\frac{p_{r}}{\sqrt{2}}+\frac{l \sqrt{\mathcal{I}}}{r}, \\
u_{a} & =\sqrt{I_{a}} \mathrm{e}^{\Phi_{a}} \quad \text { with } \quad \operatorname{Im} z>0 .
\end{aligned}
$$

These variables have the following nonvanishing Poisson brackets:

$$
\begin{aligned}
\{z, \bar{z}\} & =-\frac{l(z-\bar{z})^{2}}{2 \sqrt{2 \mathcal{I}}} \\
\left\{u_{a}, \bar{u}_{b}\right\} & =-l \delta_{a b},\left\{z, u_{a}\right\}=-u_{a} \Omega_{a} \frac{l(\bar{z}-z)}{2 \sqrt{2 \mathcal{I}}} \\
\left\{z, \bar{u}_{a}\right\} & =\bar{u}_{a} \Omega_{a} \frac{l(\bar{z}-z)}{2 \sqrt{2 \mathcal{I}}}
\end{aligned}
$$

where

$$
\Omega_{a}=\Omega_{a}(I)=\frac{\partial \sqrt{2 \mathcal{I}}}{\partial I_{a}}
$$

In these terms the generators of conformal algebra take the form

$$
\begin{aligned}
\mathcal{H}_{0} & =z \bar{z}, \\
\mathcal{D} & =\sqrt{2 \mathcal{I}\left(u_{a} \bar{u}_{a}\right)} \frac{z+\bar{z}}{l(\bar{z}-z)}, \\
\mathcal{K} & =\frac{2 \mathcal{I}\left(u_{a} \bar{u}_{a}\right)}{(l(\bar{z}-z))^{2}} .
\end{aligned}
$$

Note that the action variables $I_{a}$ complemented with the Hamiltonian form a set of Liouville integrals of the conformal mechanics (9). They have a rather simple form while being expressed via the complex variables,
$\mathcal{H}_{0}=z \bar{z}, \quad I_{a}=u_{a} \bar{u}_{a}:\left\{H_{0}, I_{a}\right\}=\left\{I_{a}, I_{b}\right\}=0$.

Let us now look for the additional integrals of motion, if any. It is easy to verify using (13) and (16) that

$$
\left\{z e^{\imath \Lambda}, \mathcal{H}_{0}\right\}=0 \quad \text { iff } \quad\{\Lambda, \sqrt{2 \mathcal{I}}\}=-1 .
$$

To get the single-valued function we impose $\Lambda \in[0,2 \pi)$. The local solutions of the above equation read

$$
\Lambda_{a}=\frac{\Phi_{a}}{\Omega_{a}}
$$

where $\Phi_{a} \in[0,2 \pi)$ is the angle variable and $I_{a}$ is given by (14). Therefore, the following local quantities are preserved and generate the set of $N-1$ additional constants of motion:

$$
M_{a}=z u_{a}^{\frac{1}{\Omega_{a}}}=z I_{a}^{\frac{1}{2 \Omega_{a}}} e^{i_{\frac{\Phi_{a}}{\Omega_{a}}}}, \quad\left\{M_{a}, \mathcal{H}_{0}\right\}=0
$$

Using (12) and (13), one can verify that the only nontrivial Poisson bracket relations among them occur between the conjugate $M_{a}$ 's,

$\left\{M_{a}, M_{b}\right\}=0, \quad\left\{M_{a}, \bar{M}_{b}\right\}=-\frac{l \delta_{a b}}{\Omega_{a}^{2}} I_{a}^{\frac{1}{\Omega_{a}}-1} \mathcal{H}_{0}$.

However, for the generic $\Omega_{a}$, the constant (19) is still not globally well defined, since $\Lambda \in\left[0,2 \pi / \Omega_{a}\right)$. To get the global solution for a certain coordinate $\Phi_{a}$, we are forced to set $\Omega_{a}$ to a rational number,

$$
\Omega_{a}=k_{a}=\frac{n_{a}}{m_{a}}, \quad m_{a}, n_{a} \in \mathbb{N} .
$$

Then, taking the $n_{a}$ th power for the locally defined conserved quantity, we get a globally defined constant of motion for the system,

$$
\mathcal{M}_{a}=M_{a}^{n_{a}}=z^{n_{a}} u_{a}^{m_{a}}=I_{a}^{\frac{m_{a}}{2}} z^{n_{a}} \mathrm{e}^{m_{a} \Phi_{a}} .
$$

Although both $M_{a}$ and $\mathcal{M}_{a}$ are complex, their absolute values are expressed via Liouville integrals and, hence, do not produce new constants of motion,

$$
\left|M_{a}\right|^{2}=\mathcal{H}_{0} I_{a}^{\frac{1}{K_{a}}}, \quad\left|\mathcal{M}_{a}\right|^{2}=\mathcal{H}_{0}^{n_{a}} I_{a}^{m_{a}} .
$$

So, we have constructed $2 N-1$ functionally independent constants of motion of the generic superintegrable conformal mechanics (9) with rational frequencies (18). Therefore, the conformal mechanics will be superintegrable provided that the angular Hamiltonian has the form (7) with rational numbers $k_{a}(21)$ and arbitrary constant $c_{0}$. 
Full symmetry algebra is given by the relations

$$
\begin{aligned}
\left\{\mathcal{M}_{a}, \overline{\mathcal{M}}_{b}\right\} & =-\imath \delta_{a b} m_{a}^{2} I_{a}^{m_{a}-1} \mathcal{H}_{0}^{n_{a}}, \\
\left\{H_{0}, \mathcal{M}_{a}\right\} & =\left\{\mathcal{M}_{a}, \mathcal{M}_{b}\right\}=0 .
\end{aligned}
$$

Note that

$$
\left\{I_{a}, \mathcal{M}_{b}\right\}=\imath \delta_{a b} M_{b}, \quad\left\{H_{0}, I_{a}\right\}=\left\{I_{a}, I_{b}\right\}=0 .
$$

As we mentioned in the Introduction, the presented formulas are applicable not only for the nonrelativistic conformal mechanics on $\mathrm{N}$-dimensional Euclidean space defined by the Hamiltonian (9) but also for the generic finite-dimensional system with conformal symmetry, including the relativistic one. A typical example of such a system is a particle moving in the near-horizon limit of an extreme black hole. Several examples of such systems were investigated by Galajinsky and his collaborators (see Ref. [20] and references therein).

\section{DEFORMED OSCILLATOR AND COULOMB SYSTEMS}

Let us extend the above consideration to the deformed $\mathrm{N}$-dimensional oscillator and Coulomb systems defined by the Hamiltonians

$\mathcal{H}_{\text {osc } / \text { Coul }}=\frac{p_{r}^{2}}{2}+\frac{\mathcal{I}}{r^{2}}+V_{\text {osc } / \text { Coul }}(r)=z \bar{z}+V_{\text {osc } / \text { Coul }}(r)$,

where

$$
\begin{gathered}
V_{\text {osc }}=\frac{\omega^{2} r^{2}}{2}=\omega^{2} \mathcal{K}=-\frac{2 \omega^{2} \mathcal{I}}{(\bar{z}-z)^{2}}, \\
V_{\text {Coul }}=-\frac{\gamma}{r}=-\frac{\gamma}{\sqrt{2 \mathcal{K}}}=-\gamma \frac{l(\bar{z}-z)}{2 \sqrt{\mathcal{I}}} .
\end{gathered}
$$

Clearly, the action variables of the angular mechanics $I_{a}$ together with the corresponding Hamiltonian define Liouville constants of motion,

$$
\left\{H_{\mathrm{osc} / \mathrm{Coul}}, I_{a}\right\}=\left\{I_{a}, I_{b}\right\}=0 .
$$

To endow these systems with the superintegrability property we choose the angular part given by (7) with rational $k_{a}$; see [7]. Below we construct the additional constants of motion and calculate their algebra for both systems in terms of complex variables (12) introduced in the previous section.

\section{A. Oscillator case}

The $2 N-2$ constants of motion of the deformed oscillator $\mathcal{H}_{\mathrm{osc}}$ in the coordinates (12) appear as

$$
\begin{aligned}
\mathcal{M}_{a}^{\mathrm{osc}} & =\left(z^{2}-\frac{2 \omega^{2} \mathcal{I}}{(\bar{z}-z)^{2}}\right)^{n_{a}} u_{a}^{2 m_{a}}, \\
\left|\mathcal{M}_{a}^{\mathrm{osc}}\right|^{2} & =\left(\mathcal{H}_{\mathrm{osc}}^{2}-2 \omega^{2} \mathcal{I}\right)^{n_{a}} I_{a}^{2 m_{a}} .
\end{aligned}
$$

Equation (29) together with (7) means that only the arguments of these complex quantities give rise to new integrals independent of the Liouville ones.

In fact, they are based on the simpler quantities $A_{a}$ and $B_{a}$, which oscillate in time with the same frequency $w$,

$$
\begin{aligned}
A_{a} & =\left(z+\frac{\omega \sqrt{2 \mathcal{I}}}{\bar{z}-z}\right) u_{a}^{\frac{1}{k_{a}}}, \\
B_{a} & =\left(z-\frac{\omega \sqrt{2 \mathcal{I}}}{\bar{z}-z}\right) u_{a}^{\frac{1}{k_{a}}}:\left\{\mathcal{H}_{\mathrm{osc}}, A_{a}\right\}=\imath \omega A_{a}, \\
\left\{\mathcal{H}_{\mathrm{osc}}, B_{a}\right\} & =-l \omega B_{a} .
\end{aligned}
$$

So, the product $A_{a} B_{b}$ is preserved,

$$
\left\{\mathcal{H}_{\mathrm{osc}}, A_{a} B_{b}\right\}=0,
$$

but is not single valued. Thus, we have to take its $n_{a}$ th power to get a well-defined constant of motion, which is precisely (29),

$$
\mathcal{M}_{a}^{\mathrm{osc}}=\left(A_{a} B_{a}\right)^{n_{a}} .
$$

Note that the reflection $\omega \rightarrow-\omega$ in the parameter space maps between $A_{a}$ and $B_{a}$. Together with the complex conjugate, they are subjected to the following rules:

$$
\begin{aligned}
\left|B_{a}\right|^{2} & =\frac{\mathcal{H}_{\mathrm{osc}}-\omega \sqrt{2 \mathcal{I}}}{\mathcal{H}_{\mathrm{osc}}+\omega \sqrt{2 \mathcal{I}}}\left|A_{a}\right|^{2}, \\
\left|A_{a}\right|^{2} & =I_{a}^{\frac{1}{k_{a}}}\left(\mathcal{H}_{\mathrm{osc}}+\omega \sqrt{2 \mathcal{I}}\right) .
\end{aligned}
$$

The complex observables $A_{a}$ and $B_{a}$ are in involution,

$$
\left\{A_{a}, A_{b}\right\}=\left\{B_{a}, B_{b}\right\}=\left\{A_{a}, B_{b}\right\}=0,
$$

so that the constants of motion (29) commute as well,

$$
\left\{\mathcal{M}_{a}^{\text {osc }}, \mathcal{M}_{b}^{\text {osc }}\right\}=0 .
$$

However, in contrast to the simplicity of the relations (25), the Poisson brackets between $\mathcal{M}_{a}^{\text {osc }}$ and $\overline{\mathcal{M}}_{b}^{\text {osc }}$ are more elaborate. They can be derived from the Poisson brackets between $A_{a}$ and $B_{a}$ and their conjugates having the following form:

$$
\begin{aligned}
& \left\{A_{a}, \bar{B}_{b}\right\}=-\frac{l \delta_{a b}}{k_{a}^{2} I_{a}} A_{a} \bar{B}_{a}, \\
& \left\{\bar{A}_{a}, B_{b}\right\}=\frac{l \delta_{a b}}{k_{a}^{2} I_{a}} \bar{A}_{a} B_{a},
\end{aligned}
$$




$$
\left\{A_{a}, \bar{A}_{b}\right\}=-\frac{2 \imath \omega A_{a} \bar{A}_{b}}{\mathcal{H}_{o s c}+\omega \sqrt{2 \mathcal{I}}}-\frac{l \delta_{a b}}{k_{a}^{2}} I_{a}^{\frac{1}{k_{a}}-1}\left(\mathcal{H}_{o s c}+\omega \sqrt{2 \mathcal{I}}\right),
$$

$$
\left\{B_{a}, \bar{B}_{b}\right\}=\frac{2 \imath \omega A_{a} \bar{A}_{b}}{\mathcal{H}_{o s c}-\omega \sqrt{2 \mathcal{I}}}-\frac{i \delta_{a b}}{k_{a}^{2}} I_{a}^{\frac{1}{k_{a}}-1}\left(\mathcal{H}_{o s c}-\omega \sqrt{2 \mathcal{I}}\right) .
$$

Hence, we have extended the holomorphic factorization formalism to the $N$ oscillator.

\section{B. Coulomb case}

The $2 N-2$ locally defined integrals of the generalized Coulomb Hamiltonian can be written in the coordinates (12) as follow:

$M_{a}^{\text {Coul }}=\left(z-\frac{l \gamma}{2 \sqrt{\mathcal{I}}}\right) u_{a}^{\frac{1}{k_{a}}}, \quad\left\{\mathcal{H}_{\text {Coul }}, M_{a}^{\text {Coul }}\right\}=0$.

As in the previous cases, only their arguments produce conserved quantities independent from the Liouville integrals (28) since

$$
\left|M_{a}^{\text {Coul }}\right|^{2}=\left(\mathcal{H}_{\text {Coul }}+\frac{\gamma^{2}}{4 \mathcal{I}}\right) I_{a}^{\frac{1}{k_{a}}}
$$

They form the following algebra, which can be verified using the Poisson brackets (13):

$$
\begin{aligned}
\left\{M_{a}^{\text {Coul }}, \bar{M}_{b}^{\text {Coul }}\right\}= & \frac{l \gamma^{2} M_{a}^{\text {Coul }} \bar{M}_{b}^{\text {Coul }}}{\sqrt{2 \mathcal{I}}\left(\gamma^{2}+4 \mathcal{I} \mathcal{H}_{\text {Coul }}\right)} \\
& -\frac{l \delta_{a b}{ }_{a}^{\frac{1}{K_{a}}-1}}{k_{a}^{2}}\left(\mathcal{H}_{\text {Coul }}+\frac{\gamma^{2}}{\sqrt{8 \mathcal{I}}}\right), \\
\left\{M_{a}^{\text {Coul }}, M_{b}^{\text {Coul }}\right\}= & 0 .
\end{aligned}
$$

Let us also present the Poisson brackets of these quantities with Liouville constants of motion

$$
\left\{I_{a}, M_{b}^{\text {Coul }}\right\}=\frac{l \delta_{a b}}{k_{b}} M_{b}^{\text {Coul }}
$$

Similar to the previous cases, we are forced to take certain powers of the local quantities (39) in order to get the valid, globally defined additional constants of motion of the deformed Coulomb problem,

$$
\mathcal{M}_{a}^{\text {Coul }}=\left(M_{a}^{\text {Coul }}\right)^{n_{a}}=\left(z-\frac{l \gamma}{2 \sqrt{\mathcal{I}}}\right)^{n_{a}} u_{a}^{m_{a}} .
$$

Their algebra can be deduced from the Poisson bracket relations (41) and (42).
So, in this section we extended the method of holomorphic factorization initially developed for the twodimensional oscillator and Coulomb system, to the superintegrable generalizations of Coulomb and oscillator systems in any dimension. For this purpose we parametrized the angular parts of these systems by action-angle variables. To our surprise, we were able to get, in these general terms, the symmetry algebra of these systems. Notice that the above formulas hold not only on the Euclidean spaces but also for the more general one, if we choose $\mathcal{I}$ to be the system with a phase space different from $T_{*} S^{N-1}$.

\section{OSCILLATOR-COULOMB CORRESPONDENCE}

As is known, the energy surface of the radial oscillator can be transformed to the energy surface of the radial Coulomb problem by transformation $\tilde{r}=\lambda r^{2}, \tilde{p}_{\tilde{r}}=p_{r} / 2 \lambda r$ where $r, p_{r}$ are the radial coordinate and momentum of the oscillator, $\tilde{r}, \tilde{p}_{\tilde{r}}$ are those of Coulomb problem, and $\lambda$ is an arbitrary positive constant number (see, e.g., [21] for the review). The extension of oscillator-Coulomb correspondence from the radial part to the whole system, as well as to its quantum counterpart, yields additional restrictions on the geometry of configuration spaces. Namely, only $N=2$, $4,8,16$-dimensional oscillators could be transformed to the Coulomb system; that is, the $N=2,5$, 9-dimensional Coulomb problem. These dimensions are distinguished due to Hopf maps $S^{1} / S^{0}=S^{1}, S^{3} / S^{1}=S^{2}, S^{7} / S^{3}=S^{4}$, which allow one to transform spherical (angular) parts of the oscillator to those of the Coulomb problem. Indeed, for the complete correspondence between the oscillator and Coulomb systems we should be able to transform the angular part of the oscillator (that is, the particle on $S^{D-1}$ ) to the angular part of the Coulomb problem, i.e., to $S^{d-1}$. Thus, the only admissible dimensions are $D=2,4,8,16$ and $d=2,3,5,9$. In the first three cases we have to reduce the initial system by $Z_{2}, U(1)$, and $S U(2)$. For the latter case, in spite of many attempts, we do not know rigorous derivation of this correspondence, due to the fact that the $S^{7}$ sphere has no Lie group structure. Respectively, in the generic case we get the extension of the two-, three-, or five-dimensional Coulomb system specified by the presence of the $Z_{2} /$ Dirac/SU(2) Yang monopole [22]. In the deformed Coulomb and oscillator problems considered in this article we do not require that the angular parts of the systems should be spheres. Hence, trying to relate these systems we are not restricted by the systems of the mentioned dimensions. Instead, we can try to relate the deformed oscillator and Coulomb systems of the same dimension and find the restrictions to the structure of their angular parts.

Below we describe this correspondence in terms of complex variables as introduced in the previous section. Through this subsection we will use "untilded" notation for 
the description of the oscillator, and "tilded" notation for the description of the Coulomb system.

The expression of the "Lobachevsky variable" (12) via radial coordinate and momentum forces one to relate the angular parts of the oscillator and Coulomb problems by the expression $\tilde{\mathcal{I}}=\mathcal{I} / 4$. The latter induces the following relations between "anglelike" variables $\Lambda, \tilde{\Lambda}: \tilde{\Lambda}=2 \Lambda$. Altogether read

$$
\begin{aligned}
& \tilde{z}=\frac{l(\bar{z}-z)}{\lambda \sqrt{\mathcal{I}}} z, \quad \tilde{\mathcal{I}}=\frac{\mathcal{I}}{4}, \\
& \tilde{\Lambda}=2 \Lambda \Leftrightarrow z=2 \sqrt{\lambda} \sqrt[4]{\tilde{\mathcal{I}}} \frac{\tilde{z}}{\sqrt{l(\overline{\tilde{z}}-\tilde{z})}}, \\
& \mathcal{I}=4 \tilde{\mathcal{I}}, \quad \Lambda=\frac{\tilde{\Lambda}}{2} .
\end{aligned}
$$

This transformation is canonical in a sense, which preserves Poisson brackets between $z, \bar{z}, \Lambda, \mathcal{I}$, and their tilded counterparts. To make the transformation canonical, we preserve the angular variables unchanged $\tilde{u}_{a}=u_{a}$, which implies the introduction of superintegrable systems with the following identification:

$$
\tilde{k}_{a}=\frac{k_{a}}{2} \Rightarrow \tilde{n}_{a}=n_{a}, \quad \tilde{m}_{a}=2 m_{a} .
$$

Then we can see that this transformation relates the energy surfaces of oscillator and Coulomb systems,

$$
\begin{aligned}
& z \bar{z}+\Omega^{2} \frac{2 \mathcal{I}}{(l(\bar{z}-z))^{2}}-E_{\mathrm{osc}} \\
& =0 \Leftrightarrow \frac{2 \lambda \sqrt{\tilde{\mathcal{I}}}}{l(\overline{\tilde{z}}-\tilde{z})}\left(\tilde{z} \tilde{\bar{z}}-\gamma \frac{l(\tilde{\bar{z}}-\tilde{z})}{2 \sqrt{\mathcal{I}}}-\tilde{\mathcal{E}}_{\text {Coul }}\right)=0,
\end{aligned}
$$

where

$$
\tilde{\gamma}=\frac{E_{\text {osc }}}{\lambda}, \quad \tilde{\mathcal{E}}_{\text {Coul }}=-\frac{2 \Omega^{2}}{\lambda^{2}} .
$$

The generators of hidden symmetries also transform one into the other on the energy surface

$$
\mathcal{M}_{(a) \text { osc }}=(\imath \lambda \sqrt[4]{2 \tilde{\mathcal{I}}})^{n_{a}} \mathcal{M}_{(a) \text { Coul }}
$$

Finally, let us write down the relation between generators of conformal symmetries defined on tilded and untilded spaces,

$$
\mathcal{H}_{0}=\lambda \tilde{\mathcal{H}}_{0} \sqrt{2 \tilde{\mathcal{K}}}, \quad \mathcal{D}=2 \tilde{\mathcal{D}}, \quad \mathcal{K}=\frac{2 \sqrt{2 \tilde{\mathcal{K}}}}{\lambda} .
$$

In this section we transformed the deformed oscillator into the deformed Coulomb problem, preserving intact angular coordinates. Performing proper transformations of angular parts of the oscillator, including its reduction, we can get a variety of superintegrable deformations of the Coulomb problem. However, they will belong to the same class of systems under consideration, since the latter are formulated in most general, action-angle variables and terms.

\section{SPHERICAL AND PSEUDOSPHERICAL GENERALIZATIONS}

Oscillator and Coulomb systems admit superintegrable generalizations to $\mathrm{N}$-dimensional spheres and two-sheet hyperboloids (pseudospheres), which are given by the Hamiltonians [5]

$$
\begin{aligned}
& \mathbb{S}^{N}: \mathcal{H}_{V}=\frac{p_{\chi}^{2}}{2 r_{0}^{2}}+\frac{\mathcal{I}}{r_{0}^{2} \sin ^{2} \chi}+V(\tan \chi), \\
& \mathbb{\boxplus}^{N}: \mathcal{H}_{V}=\frac{p_{\chi}^{2}}{2 r_{0}^{2}}+\frac{\mathcal{I}}{r_{0}^{2} \sinh ^{2} \chi}+V(\tanh \chi)
\end{aligned}
$$

with the potentials

$$
\begin{aligned}
\mathbb{S}^{N}: V_{\text {osc }}(\tan \chi) & =\frac{r_{0}^{2} \omega^{2} \tan ^{2} \chi}{2}, \\
V_{\text {Coul }}(\tan \chi) & =-\frac{\gamma}{r_{0}} \cot \chi, \\
\mathbb{H}^{N}: V_{\text {osc }}(\tanh \chi) & =\frac{r_{0}^{2} \omega^{2} \tanh ^{2} \chi}{2}, \\
V_{\text {Coul }}(\tanh \chi) & =-\frac{\gamma}{r_{0}} \operatorname{coth} \chi .
\end{aligned}
$$

Here $\mathcal{I}$ is a quadratic Casimir element of the orthogonal algebra $s o(N)$. To get integrable deformations of these systems, we replace it, as in the Euclidean case, by some integrable (angular) Hamiltonian depending on the action variables [6]. The particular angular Hamiltonian (7) defines superintegrable systems as in the flat case. About a decade ago the so-called $\kappa$-dependent formalism was developed [23] where the oscillator and Coulomb systems on the plane and on the two-dimensional sphere and hyperboloid were described in the unified way.

We introduce, following those papers,

$$
\begin{aligned}
T_{\kappa} & =\frac{S_{\kappa}}{C_{\kappa}} \text { with } \\
C_{\kappa}(x) & = \begin{cases}\cos \sqrt{\kappa} x & \kappa>0, \\
1 & \kappa=0, \\
\cosh \sqrt{-\kappa} x & \kappa<0,\end{cases} \\
S_{\kappa}(x) & = \begin{cases}\frac{\sin \sqrt{\kappa} x}{\sqrt{\kappa}} & \kappa>0, \\
x & \kappa=0, \\
\frac{\sinh \sqrt{-\kappa} x}{\sqrt{-\kappa}} & \kappa<0,\end{cases}
\end{aligned}
$$


where the parameter $\kappa$ in the two-dimensional case coincides with the curvature of the (pseudo)sphere,

$$
\mathbb{S}^{N}: \kappa=\frac{1}{r_{0}^{2}}, \quad \mathbb{\llbracket}^{N}: \kappa=-\frac{1}{r_{0}^{2}} .
$$

The case $\kappa= \pm 1$ corresponds to a unit sphere/pseudosphere. For $\kappa \neq 0$ we identify

$$
x=r_{0} \chi=\frac{\chi}{\sqrt{\kappa}}, \quad p_{x}=\frac{p_{\chi}}{r_{0}}=\sqrt{\kappa} p_{\chi} .
$$

The holomorphic factorization approach to twodimensional systems was combined with $\kappa$-dependent formalism by Ranada. Let us show that it can be straightly extended to any dimension. For this purpose we introduce a (pseudo)spherical analog of $z, \bar{z}$ coordinates and obtain their Poisson bracket,

$$
\begin{aligned}
z & =\sqrt{|\kappa|} \frac{p_{\chi}}{\sqrt{2}}+\frac{l \sqrt{\mathcal{I}}}{T_{\kappa}}, \\
\{\bar{z}, z\} & =\frac{l(z-\bar{z})^{2}}{2 \sqrt{2 \mathcal{I}}}-\imath \kappa \sqrt{2 \mathcal{I}} .
\end{aligned}
$$

The Poisson brackets among $z, u_{a}$, and $\bar{u}_{a}$ remain unchanged [see relations (13)].

In these terms the $\kappa$-deformed Hamiltonian reads

$$
\begin{aligned}
\mathcal{H}_{\text {osc } / \text { Coul }} & =\mathcal{H}_{0}+V_{\text {osc } / \text { Coul }}, \\
\mathcal{H}_{0} & =\frac{p_{r}^{2}}{2}+\frac{\mathcal{I}}{S_{\kappa}^{2}}+\kappa \mathcal{I}=z \bar{z}+\kappa \mathcal{I},
\end{aligned}
$$

where using (53), [23], (55), (56), the oscillator and Coulomb potentials on sphere (51) can be expressed as follows:

$$
\begin{aligned}
V_{\mathrm{osc}} & =\frac{\omega^{2} T_{\kappa}^{2}}{2}=-\frac{2 \omega^{2} \mathcal{I}}{(\bar{z}-z)^{2}}, \\
V_{\mathrm{Coul}} & =-\frac{\gamma}{T_{\kappa}}=-l \gamma \frac{\bar{z}-z}{2 \sqrt{\mathcal{I}}} .
\end{aligned}
$$

The (local and global) constants of motion and related quantities have the same expressions in terms of $z, \bar{z}$ as in the flat case, with the Hamiltonians shifted in agreement with (57)

$$
\mathcal{H} \rightarrow \mathcal{H}-\kappa \mathcal{I}
$$

For the free system on sphere, $\mathcal{H}_{0}$, most of the Poisson brackets among the integrals survive from the flat case [see relations (20), (24), and (25)]. The only brackets that acquire extra $\kappa$-dependent terms are

$$
\begin{aligned}
\left\{M_{a}, \bar{M}_{b}\right\} & =\left(\frac{\imath \kappa \sqrt{2 \mathcal{I}}}{\mathcal{H}_{0}-\kappa \mathcal{I}}-\frac{\imath \delta_{a b}}{k_{a}^{2} I_{a}}\right) M_{a} \bar{M}_{b} \\
& =-\frac{\imath \delta_{a b}}{k_{a}^{2}} I_{a}^{\frac{1}{k_{a}}-1}\left(\mathcal{H}_{0}-\kappa \mathcal{I}\right)+\frac{\imath \kappa \sqrt{2 \mathcal{I}}}{\mathcal{H}_{0}-\kappa \mathcal{I}} M_{a} \bar{M}_{b},
\end{aligned}
$$

$$
\left\{\mathcal{M}_{a}, \overline{\mathcal{M}}_{b}\right\}=\imath\left(\frac{\kappa n_{a} n_{b} \sqrt{2 \mathcal{I}}}{\mathcal{H}_{0}-\kappa \mathcal{I}}-\frac{m_{a}^{2} \delta_{a b}}{I_{a}}\right) \mathcal{M}_{a} \overline{\mathcal{M}}_{b}
$$

Let us also write down the deformation of conformal algebra (1)

$$
\begin{aligned}
\left\{\mathcal{H}_{0}, \mathcal{D}\right\} & =2\left(\mathcal{H}_{0}-\kappa \mathcal{I}\right)(1+2 \kappa \mathcal{K}), \\
\left\{\mathcal{H}_{0}, \mathcal{K}\right\} & =\mathcal{D}(1+2 \kappa \mathcal{K}), \\
\{\mathcal{D}, \mathcal{K}\} & =2 \mathcal{K}(1+2 \kappa \mathcal{K}) .
\end{aligned}
$$

For the Coulomb problem on the sphere, the Poisson brackets between the local integrals (42) remain unaffected, while the relations (41) undergo a similar modification,

$$
\begin{aligned}
\left\{M_{a}^{\text {Coul }}, \bar{M}_{b}^{\text {Coul }}\right\}= & {\left[\frac{\imath \sqrt{2 \mathcal{I}}\left(\frac{\gamma^{2}}{4 \mathcal{I}^{2}}+\kappa\right)}{\mathcal{H}_{\text {Coul }}-\kappa \mathcal{I}+\frac{\gamma^{2}}{4 \mathcal{I}^{2}}}-\frac{\imath \delta_{a b}}{k_{a}^{2} I_{a}}\right] M_{a}^{\text {Coul }} \bar{M}_{b}^{\text {Coul }} } \\
= & t \sqrt{2 \mathcal{I}}\left(\frac{\gamma^{2}}{4 \mathcal{I}^{2}}+\kappa\right) \frac{M_{a}^{\text {Coul }} \bar{M}_{b}^{\text {Coul }}}{\mathcal{H}_{\text {Coul }}-\kappa \mathcal{I}+\frac{\gamma^{2}}{4 \mathcal{I}^{2}}} \\
& -\frac{\imath \delta_{a b}}{k_{a}^{2}} I_{a}^{\frac{1}{k_{a}}-1}\left(\mathcal{H}_{\text {Coul }}-\kappa \mathcal{I}+\frac{\gamma^{2}}{4 \mathcal{I}^{2}}\right) \cdot
\end{aligned}
$$

Consider now the spherical system (50) with the oscillator potential. Line for the flat case, the integrals of motion are based on the simpler local quantities $A$ and $B$,

$$
\begin{aligned}
A_{a} & =\left(z+\frac{\imath \omega T_{\kappa}}{\sqrt{2}}\right) u_{a}^{\frac{1}{k_{a}}}, \\
B_{a} & =\left(z-\frac{l \omega T_{\kappa}}{\sqrt{2}}\right) u_{a}^{\frac{1}{k_{a}}}, \\
\mathcal{M}_{a}^{\text {osc }} & =\left(A_{a} B_{a}\right)^{n_{a}},
\end{aligned}
$$

which evolve in time under the following rule:

$$
\begin{aligned}
& \left\{\mathcal{H}_{\mathrm{osc}}, A_{a}\right\}=\imath \omega\left(1+\kappa T_{\kappa}^{2}\right) A_{a}, \\
& \left\{\mathcal{H}_{\mathrm{osc}}, B_{a}\right\}=-\imath \omega\left(1+\kappa T_{\kappa}^{2}\right) B_{a} .
\end{aligned}
$$

They are $\kappa$ deformations of the harmonic oscillating quantities (30) and (32) in the flat case. Unlike them, they do not oscillate harmonically, but the product $A_{a} B_{b}$ is still preserved.

The Poisson brackets between local quantities can be calculated explicitly giving rise to $\kappa$ deformations of the relations (36), (37), and (38), 


$$
\begin{aligned}
\left\{A_{a}, B_{b}\right\}= & -\frac{\imath \kappa \omega T_{\kappa}^{2}}{z^{2}+\frac{\omega^{2} T_{\kappa}^{2}}{2}} A_{a} B_{b}, \\
\left\{A_{a}, \bar{B}_{b}\right\}= & -\frac{\imath \delta_{a b}}{k_{a}^{2} I_{a}} A_{a} \bar{B}_{a}+\frac{\imath \kappa \sqrt{2 \mathcal{I}} A_{a} \bar{A}_{b}}{\mathcal{H}_{\mathrm{osc}}-\kappa \mathcal{I}+\omega \sqrt{2 \mathcal{I}}}, \\
\left\{A_{a}, \bar{A}_{b}\right\}= & \iota \frac{\kappa\left(\sqrt{2 \mathcal{I}}-2 \omega T_{\kappa}\right)-2 \omega}{\mathcal{H}_{\mathrm{osc}}-\kappa \mathcal{I}+\omega \sqrt{2 \mathcal{I}}} A_{a} \bar{A}_{b} \\
& -\frac{l \delta_{a b} \frac{1}{k_{a}}-1}{k_{a}^{2}}\left(\mathcal{H}_{\mathrm{osc}}-\kappa \mathcal{I}+\omega \sqrt{2 \mathcal{I}}\right), \\
\left\{B_{a}, \bar{B}_{b}\right\}= & \iota \frac{\kappa\left(\sqrt{2 \mathcal{I}}+2 \omega T_{\kappa}\right)+2 \omega}{\mathcal{H}_{\mathrm{osc}}-\kappa \mathcal{I}-\omega \sqrt{2 \mathcal{I}}} A_{a} \bar{A}_{b} \\
& -\frac{l \delta_{a b} \frac{1}{k_{a}}-1}{k_{a}^{2}}\left(\mathcal{H}_{\mathrm{osc}}-\kappa \mathcal{I}-\omega \sqrt{2 \mathcal{I}}\right) .
\end{aligned}
$$

The Poisson brackets between the true integrals of motion $\mathcal{M}_{a}^{\text {osc }}, \mathcal{M}_{a}^{\text {Coul }}$ and their conjugates are based on the local brackets (63), (66), (67), (68) and can easily be obtained.

\section{EXAMPLE OF SPHERICAL PART OF HIGHER- ORDER SUPERINTEGRABLE SYSTEM WITH SEPARATION OF VARIABLES}

In previous sections we extended the holomorphic factorization approach to higher-dimensional superintegrable systems with oscillator and Coulomb potentials, including those on spheres and hyperboloids. For this purpose we separated the radial and angular variables in these systems. Then we combined the radial coordinate and momentum in a single complex coordinate parametrizing the Klein model of Lobachevsky space, and we combined angular coordinates and their conjugated momenta in complex coordinates by the use of action-angle variables. However, action-angle variables are not in common use in present math-physical society, and their explicit expressions are not common even for such textbook models as the oscillator and Coulomb problems.

For clarifying the relation of the above formulations of constants of motion with their conventional representations, first present the action-angle variables of the angular part(s) of nondeformed, oscillator, and Coulomb systems (on Euclidean space, sphere, and hyperboloids). Its Hamiltonian is given by the quadratic Casimir element of $s o(N)$ algebra on the $(N-1)$ sphere, $\mathcal{I}=L_{N}^{2} / 2$. It can be decomposed by the eigenvalues of the embedded $S O(a)$ angular momenta defining the action variables $I_{a}$. For the details of derivation of their explicit expressions, for those of conjugated angle variables we refer to the appendix in Ref. [6]. The action variables are given by the expressions

$$
\begin{aligned}
I_{a} & =\sqrt{j_{a+1}}-\sqrt{j_{a}}, \\
\text { where } j_{a+1} & =p_{a}^{2}+\frac{j_{a}}{\sin ^{2} \theta_{a}}, \\
j_{0} & =0, \quad a=1, \ldots, N-1 .
\end{aligned}
$$

This gives rise to the angular Hamiltonian that belongs to the family (7)

$$
\mathcal{I}=\frac{1}{2}\left(\sum_{a=1}^{N-1} I_{a}\right)^{2}
$$

Its substitution into the Hamiltonians (26) and (50) leads to well-known oscillator and Coulomb systems on the Euclidean spaces, spheres, and hyperboloids.

The expressions for angle variables are more complicated,

$$
\begin{aligned}
\Phi_{a} & =\sum_{l=a}^{N-1} a_{l}+\sum_{l=a+1}^{N-1} b_{1}, \\
\text { where } a_{l} & =\arcsin \sqrt{\frac{j_{l+1}}{j_{l+1}-j_{l}}} \cos \theta_{l}, \\
b_{l} & =\arctan \frac{\sqrt{j_{l}} \cos \theta_{l}}{p_{l} \sin \theta_{l}} .
\end{aligned}
$$

Direct transformations give the following expressions for $u_{a}$ coordinates:

$$
u_{a}=\sqrt{\sqrt{j_{a+1}}-\sqrt{j_{a}}} \mathrm{e}^{l a_{a}} \prod_{l=a+1}^{N-1} \mathrm{e}^{\mathrm{l}^{\left(a_{l}+b_{l}\right)}}
$$

with

$$
\begin{aligned}
\mathrm{e}^{l a_{l}} & =\frac{p_{l} \sin \theta_{l}+l \sqrt{j_{l+1}} \cos \theta_{l}}{\sqrt{j_{l+1}-j_{l}}}, \\
\mathrm{e}^{l b_{l}} & =\frac{p_{l} \sin \theta_{l}+l \sqrt{j_{l}} \cos \theta_{l}}{\sqrt{j_{l+1}-j_{l}} \sin \theta_{l}} .
\end{aligned}
$$

With these expressions at hand we can express the "holomorphic representation" of constants of motion via initial coordinates. In the two-dimensional case it has a transparent relation with conventional representations of hidden constants of motion, like the Fradkin tensor (for the oscillator) and the Runger-Lenz vector (for the Coulomb problem) [17]. In the higher dimensional cases the relation of these two representations is more complicated.

This construction could easily be modified to the system whose Hamiltonian is given in the angle variables by the generic expression (7). We define it by the recurrence relation 
$\mathcal{I} \equiv \frac{1}{2} j_{N}, \quad j_{a}=p_{a-1}^{2}+\frac{j_{a-1}}{\sin ^{2} k_{a-1} \theta_{a-1}}$,

$a=1, \ldots, N-1, \quad j_{0}=c_{0}$.

It describes particle moving on the space (spherical segment) equipped with the diagonal metric

$d s^{2}=g_{l l}\left(d \theta_{l}\right)^{2}, \quad g_{N-1 . N-1}=1, \quad g_{l l}=\prod_{m=l}^{N-1} \sin ^{2} k_{m} \theta_{m}$

and interacting with the potential field

$$
U=\frac{c_{0}}{\prod_{l=1}^{N-1} \sin ^{2} k_{l} \theta_{l}}
$$

Redefining the angles, $\theta_{a} \rightarrow \theta_{a} / k_{a}$, we can represent the above metric in the form

$$
d s^{2}=\frac{1}{k_{a}^{2}} \prod_{a=1}^{N-1} \sin ^{2} \theta_{a}\left(d \theta_{a}\right)^{2}
$$

It is obvious that the functions $j_{k}(\theta, p)$ define commuting constants of motions of the system. Similar to the derivation given in the appendix of Ref. [6], we can use an action-angle variable formulation and find that the Hamiltonian is given by the expression (7). The action variables are related to the initial ones by the expressions

$$
\begin{aligned}
I_{a} & =\frac{1}{2 \pi} \int_{\theta_{\max }}^{\theta_{\min }} \sqrt{j_{a+1}-\frac{j_{a}}{\sin ^{2} k_{a} \theta_{a}}} d \theta_{a} \\
& =\frac{\sqrt{j_{a+1}}-\sqrt{j_{a}}}{k_{a}} \Rightarrow j_{a} \\
& =\left(\sum_{a=1}^{N-1} k_{a} I_{a}+c_{0}\right)^{2} .
\end{aligned}
$$

The angle variables read

$$
\begin{aligned}
\Phi_{a} & =\sum_{l=a}^{N-1} \frac{k_{a}}{k_{l}} a_{l}+\sum_{l=a+1}^{N-1} \frac{k_{a}}{k_{l}} b_{l}, \\
a_{l} & =\arcsin \sqrt{\frac{j_{l+1}}{j_{l+1}-j_{l}}} \cos k_{l} \theta_{l}, \\
b_{l} & =\arctan \frac{\sqrt{j_{l}} \cos \theta_{l}}{p_{l} \sin k_{l} \theta_{l}} .
\end{aligned}
$$

Thus,

$$
\begin{aligned}
u_{a}= & \frac{1}{k_{a}} \sqrt{\sqrt{j_{a+1}}-\sqrt{j_{a}}} \\
& \times \prod_{l=a}^{N-1}\left(\frac{p_{l} \sin k_{l} \theta_{l}+l \sqrt{j_{l+1}} \cos k_{l} \theta_{l}}{\sqrt{j_{l+1}-j_{l}}}\right)^{\frac{k_{a}}{k_{l}}} \\
& \times \prod_{l=a+1}^{N-1}\left(\frac{p_{l} \sin k_{l} \theta_{l}+l \sqrt{j_{l}} \cos k_{l} \theta_{l}}{\sqrt{j_{l+1}-j_{l}} \sin \theta_{l}}\right)^{\frac{k_{a}}{k_{l}}} .
\end{aligned}
$$

Hence, we constructed the superintegrable system with higher order constants of motion, which admits separation of variables. Since the classical spectrum of its angular part is isospectral with the "angular Calogero model," we can state that they become, under the appropriate choice of constants $k_{i}$ and $c_{0}$, canonically equivalent with the angular part of the rational Calogero model [8]. In fact, this means the equivalence of these two systems. However, we cannot present explicit mapping of one system to other.

\section{CONCLUDING REMARKS}

In this work we investigated superintegrable deformations of oscillator and Coulomb problems separating their radial and angular parts, where the latter was described in terms of action-angle variables. We encoded phase space coordinates in the complex ones: the complex coordinate $z$ involved radial variables parametrizing the Klein model of the Lobachevsky plane and complex coordinates $u_{a}$ encoding action-angle variables of the angular part. Then we combined the whole set of constants of motion (independent from the Hamiltonian) in $N-1$ holomorphic functions $\mathcal{M}_{a}$, generalizing the so-called holomorphic factorization earlier developed for two-dimensional generalized oscillator and Coulomb systems. Then we presented their algebra, which among nontrivial relations possesses chirality property $\left\{\mathcal{M}_{a}, \mathcal{M}_{a}\right\}=0$. Hence, the presented representation can obviously be considered as a classical trace of "quantum factorization" of the respective Hamiltonian. It seems that it could be used for the construction of supersymmetric extensions of these systems. The lack of given representation is shown by the use of the action-angle formulation of the angular parts of the original systems.

In this context we should mention the earlier work [24], where symmetries of the angular parts of conformal mechanics (and those with additional oscillator potential) were related to the symmetries of the whole system by the use of coordinate $z$ and conformal algebra generators (15). That study was done in most general terms, without referring to action-angle variables and to a specific form of the angular part. Quantum mechanical aspects were also considered there. Hence, it seems to be natural to combine these two approaches: first, exclude the action-angle argument from present formulations, and second, use presented constructions for the quantum considerations of systems, in particular, for the construction of spectrum and wave 
functions within Foperator approach. We are planning to present this elsewhere.

\section{ACKNOWLEDGMENTS}

This work was partially supported by the Armenian State Committee of Science Grants No. 15RF-039 and
No. 15T-1C367 and by Grant No. mathph-4220 of the Armenian National Science and Education Fund based in New York (ANSEF). It was done within ICTP programs NET68 and OEA-AC-100 and within the program of Regional Training Network on Theoretical Physics sponsored by Volkswagenstiftung Contract No. 86260.
[1] F. Calogero, Solution of a three-body problem in onedimension, J. Math. Phys. (N.Y.) 10, 2191 (1969); Solution of the one-dimensional N-body problems with quadratic and/or inversely quadratic pair potentials, J. Math. Phys. (N.Y.) 12, 419 (1971); J. Moser, Three integrable Hamiltonian systems connected with isospectral deformations, Adv. Math. 16, 197 (1975).

[2] M. Olshanetsky and A. Perelomov, Classical integrable finite dimensional systems related to Lie algebras, Phys. Rep. 71, 313 (1981); Quantum integrable systems related to Lie algebras, Phys. Rep. 94, 313 (1983).

[3] S. Wojciechowski, Superintegrability of the CalogeroMoser system, Phys. Lett. 95A, 279 (1983).

[4] V. de Alfaro, S. Fubini, and G. Furlan, Conformal invariance in quantum mechanics, Nuovo Cimento Soc. Ital. Fis. 34A, 569 (1976).

[5] P. W. Higgs, Dynamical symmetries in a spherical geometry. 1, J. Phys. A 12, 309 (1979); H. I. Leemon, Dynamical symmetries in a spherical geometry. 2, J. Phys. A 12, 489 (1979).

[6] T. Hakobyan, O. Lechtenfeld, A. Nersessian, A. Saghatelian, and V. Yeghikyan, Integrable generalizations of oscillator and Coulomb systems via action-angle variables, Phys. Lett. A 376, 679 (2012).

[7] T. Hakobyan, O. Lechtenfeld, and A. Nersessian, Superintegrability of generalized Calogero models with oscillator or Coulomb potential, Phys. Rev. D 90, 101701 (2014).

[8] M. Feigin, O. Lechtenfeld, and A. Polychronakos, The quantum angular Calogero-Moser model, J. High Energy Phys. 07 (2013) 162.

[9] T. Hakobyan and A. Nersessian, Runge-Lenz vector in Calogero-Coulomb problem, Phys. Rev. A 92, 022111 (2015).

[10] F. Correa, T. Hakobyan, O. Lechtenfeld, and A. Nersessian, Spherical Calogero model with oscillator/Coulomb potential: Quantum case, Phys. Rev. D 93, 125009 (2016); Spherical Calogero model with oscillator/Coulomb potential: Classical case, Phys. Rev. D 93, 125008 (2016).

[11] T. Hakobyan and A. Nersessian, Integrability and separation of variables in Calogero-Coulomb-Stark and two-center Calogero-Coulomb systems, Phys. Rev. D 93, 045025 (2016).

[12] F. Tremblay, A. V. Turbiner, and P. Winternitz, An infinite family of solvable and integrable quantum systems on a plane, J. Phys. A 42, 242001 (2009).

[13] S. Post and P. Winternitz, An infinite family of superintegrable deformations of the Coulomb potential, J. Phys. A 43, 222001 (2010).
[14] E. G. Kalnins, K. M. Kress, and W. Miller, Superintegrability and higher order constants for quantum systems, J. Phys. A 43, 265205 (2010).

[15] O. Lechtenfeld, A. Nersessian, and V. Yeghikyan, Actionangle variables for dihedral systems on the circle, Phys. Lett. A 374, 4647 (2010).

[16] M. F. Ranada, The Tremblay-Turbiner-Winternitz system on spherical and hyperbolic spaces: Superintegrability, curvature-dependent formalism and complex factorization, J. Phys. A 47, 165203 (2014); A new approach to the higher order superintegrability of the Tremblay-Turbiner-Winternitz system, J. Phys. A 45, 465203 (2012); Higher order superintegrability of separable potentials with a new approach to the Post-Winternitz system, J. Phys. A 46, 125206 (2013).

[17] T. Hakobyan, A. Nersessian, and H. Shmavonyan, Lobachevsky geometry in TTW and PW systems, arXiv:1512.07489.

[18] T. Hakobyan and A. Nersessian, Lobachevsky geometry of (super)conformal mechanics, Phys. Lett. A 373, 1001 (2009); C. Burdik and A. Nersessian, Remarks on multi-dimensional conformal mechanics, SIGMA 5, 004 (2009).

[19] T. Hakobyan, A. Nersessian, and V. Yeghikyan, Cuboctahedric Higgs oscillator from the Calogero model, J. Phys. A 42, 205206 (2009); T. Hakobyan, S. Krivonos, O. Lechtenfeld, and A. Nersessian, Hidden symmetries of integrable conformal mechanical systems, Phys. Lett. A 374, 801 (2010); T. Hakobyan, O. Lechtenfeld, A. Nersessian, and A. Saghatelian, Invariants of the spherical sector in conformal mechanics, J. Phys. A 44, 055205 (2011); T. Hakobyan, O. Lechtenfeld, and A. Nersessian, The spherical sector of the Calogero model as a reduced matrix model, Nucl. Phys. B858, 250 (2012); F. Correa and O. Lechtenfeld, The tetrahexahedric angular Calogero model, J. High Energy Phys. 10 (2015) 191.

[20] S. Bellucci, A. Galajinsky, E. Ivanov, and S. Krivonos, AdS (2)/CFT(1), canonical transformations and superconformal mechanics, Phys. Lett. B 555, 99 (2003); A. Galajinsky, Particle dynamics near extreme Kerr throat and supersymmetry, J. High Energy Phys. 11 (2010) 126; A. Galajinsky and K. Orekhov, $N=2$ superparticle near horizon of extreme Kerr-Newman-AdS-dS black hole, Nucl. Phys. B850, 339 (2011); A. Galajinsky, A. Nersessian, and A. Saghatelian, Superintegrable models related to near horizon extremal Myers-Perry black hole in arbitrary dimension, J. High Energy Phys. 06 (2013) 002; A. Galajinsky and K. 
Orekhov, On the near horizon rotating black hole geometries with NUT charges, Eur. Phys. J. C 76, 477 (2016).

[21] V. Ter-Antonian, Dyon oscillator duality, arXiv:quant-ph/ 0003106; A. Nersessian and V. M. Ter-Antonian, Anyons, monopole and Coulomb problem, Yad. Fiz. 61, 1868 (1998) [Phys. At. Nucl. 61, 1756 (1998)].

[22] A. Nersessian, V. Ter-Antonian, and M. M. Tsulaia, A note on quantum Bohlin transformation, Mod. Phys. Lett. A 11, 1605 (1996); A. Nersessian and V. Ter-Antonian, "Charge dyon', system as the reduced oscillator, Mod. Phys. Lett. A 09, 2431 (1994); L. G. Mardoyan, A. N. Sisakian, and V. M. Ter-Antonian, Hidden symmetry of the Yang-Coulomb system, Mod. Phys. Lett. A 14, 1303 (1999).
[23] P. Dombrowski and J. Zitterbarth, On the planetary motion in the 3-dim standard spaces of constant curvatur, Demonstratio mathematica 24, 375 (1991); A. Ballesteros, F. J. Herranz, M. A. del Olmo, and M. Santander, Quantum structure of the motion groups of the two-dimensional Cayley-Klein geometries, J. Phys. A: Math. Gen. 26, 5801 (1993); M. F. Rañada and M. Santander, Superintegrable systems on the two-dimensional sphere S2 and the hyperbolic plane H2, J. Math. Phys. 40, 5026 (1999).

[24] T. Hakobyan, D. Karakhanyan, and O. Lechtenfeld, The structure of invariants in conformal mechanics, Nucl. Phys. B886, 399 (2014). 